\title{
Marroquíes en España: un análisis por género de los determinantes en las migraciones familiares
}

\author{
Moroccans in Spain: an analysis by gender of the \\ determinant factors on family migration \\ Marocains en l'Espagne: une analyse par genre des \\ déterminants dans les migrations familiales
}

\author{
Carolina Montoro-Gurich ${ }^{1}$
}

\section{RESUMEN}

El objetivo de este trabajo es analizar, desde la perspectiva de género, los factores incidentes en el calendario migratorio a España de personas marroquíes casadas. Para ello se emplea la información proveniente de una encuesta etnográfica ( $\mathrm{n}=262$ sujetos) aplicada en 2013 a población marroquí residente en Navarra, y se estiman modelos de regresión logística para predecir qué variables tienen más influencia en la reagrupación familiar acelerada de los sujetos. Los resultados muestran cómo en ambos modelos, masculino y femenino, el determinante más relevante es que la mujer trabaje en el mismo año de la reagrupación, seguido del lapso de tiempo transcurrido entre la reagrupación y 2008. Además, en el modelo masculino, es importante la consideración de si la mujer puede o no trabajar fuera de casa mientras que en el femenino, son significativas las variables relacionadas con capital social como: edad del cónyuge, hijos pequeños o jefatura del hogar. La conclusión es que la reagrupación familiar temprana en los marroquíes refleja una realidad marcada por motivaciones económicas y, también, por diferencias de género.

\footnotetext{
1 Departamento de Historia, Historia de Arte y Geografía, Universidad de Navarra. cmontoro@unav.es ORCID iD: http://orcid.org/0000-0002-7788-0240
} 
Palabras Clave: determinantes migraciones; migración familiar; género; marroquíes; España.

\begin{abstract}
This work analyses the most influential factors over the migratory calendar of Moroccan married people moving to Spain from the gender-related perspective. It uses data coming from an ethnographic survey (=262 individuals) applied in 2013 to Moroccan population in Navarre. Logistic regression models are estimated to forecast which variables have more influence on the accelerated family reunification of such individuals. Results show that in both models, male and female, the most relevant determinant is the fact about if women do work in the same year of the reunification, followed by the time interval between the reunification and year 2008. Moreover, in the male model, the opinion of men about female occupation outside the home is very important, while in the female one there are remarkable variables related to social capital such as: spouse's age, having small children and being the head of householdor not. The conclusion is that early family reunification in Moroccans reveals a reality wich is conditioned both by economic motivations and gender differences.
\end{abstract}

KEY WORDS: Determinants migrations; family migration; gender; Moroccans; Spain.

\title{
RÉSUMÉ
}

Ce travail analyse dans une perspective de genre les facteurs qui ont une incidence sur le calendrier migratoire vers l'Espagne des personnes marocaines mariées. En employant l'enquête ethnographique (n=262 individus) appliquée en 2014 à la population marocaine résidante en Navarre, des modèles de régression logistique ont été estimées pour prédire quelles variables ont le plus d'influence sur la réunification familiale accélérée des individus étudiés. Les résultats montrent que dans les deux modèles, masculin et féminin, le déterminant le plus remarquable c'est le fait que la femme travaille dans la même année de la réunification, suivi du laps de temps entre la réunification et 2008. De plus, dans le modèle masculin, l'opinion de l'homme sur l'occupation de la femme en dehors de son foyer est significative, et dans le féminin, les variables significatives concernent le capital social, comme: l'âge du conjoint, avoir de petits enfants et être, ou non, le chef de famille. En conclusión, la réunification familiale précoce entre les Marocains relève une réalité marquée par des motivations économiques et aussi, différences de genre.

Mots-ClÉs: déterminants migrations; migration familiale; genre; Marocains; Espagne.

Cómo citar este artículo/Citation: Montoro-Gurich, Carolina (2017): "Marroquíes en España: un análisis por género de los determinantes en las migraciones familiares", Estudios Geográficos, LXXVIII/283, pp. 445-464. 


\section{INTRODUCCIÓN}

Massey y Espinoza Higgins señalan en un trabajo reciente (2014), cómo Marruecos es un buen ejemplo de lo que Skeldon (1997) denomina «naciónfrontera laboral», porque presenta un estado de desarrollo económico y de infraestructuras intermedio que, por un lado facilita la movilidad y búsqueda de oportunidades de los individuos fuera de las comunidades locales y, por otro, no es capaz de ofrecer empleo a todos los ciudadanos, con lo que los impulsa a migrar fuera de sus fronteras.

La historia migratoria de Marruecos con destino a Europa es larga, y especialmente intensa desde los años 60 del siglo XX, cuando se firman acuerdos migratorios con Francia y Alemania (1963), Bélgica (1964) y Holanda (1969) (de Haas, 2007). Importantes contingentes de hombres marroquíes se dirigían a estos países para trabajar en las minas, la metalurgia y la construcción. Con la crisis del petróleo de 1973 las potencias europeas se plantean como objetivo la «inmigración cero» y la reunificación familiar permanece como única vía de entrada en Europa (Cebolla Boado y Requena Díez de Revenga, 2009). Desde entonces los emigrantes marroquíes diversificaron sus destinos, aprovechando los vacíos legales y de control de la migración, y España, junto con Italia, se incorpora al grupo de países que reciben población marroquí poco cualificada.

Desde la década de los 90 España experimenta una importante transformación, convirtiéndose en un país de inmigración con una rapidez y una intensidad sin precedentes en Europa (Cebolla Boado y González Ferrer, 2008: 16). De entre todos los flujos con destino a España, los marroquíes son los que más van a crecer —entre 1990 y 2005 en un 673\% (de Haas, 2005) — y se convierten en uno de los principales colectivos de inmigrantes: alrededor del $12 \%$ de los extranjeros que residen en España son de origen marroquí (Massey y Espinoza Higgins, 2014). El perfil dominante del inmigrante marroquí en España en estos primeros años va a ser el masculino, y su motivo para migrar, el económico. Las mujeres se sumaron a la migración pasado un tiempo, mayoritariamente como resultado de la reagrupación familiar, hecho que ha favorecido un mayor equilibrio por sexos del efectivo marroquí inmigrante en España y da fe de ser una migración que ha entrado en la fase de maduración del ciclo migratorio (Izquierdo Escribano y León-Alfonso, 2008).

Por otro lado, en los últimos años se ha detectado una diversificación creciente de los perfiles migratorios femeninos, tanto desde el punto de vista de las características socio-demográficas de las migrantes como desde el de los motivos que impulsaron la migración (Montoro Gurich, 2013; Reinaldo 
Espinosa, 2007; Soriano Miras, 2006). Se aprecia una presencia creciente de mujeres migrantes solas y, también, una mayor influencia de la mujer casada en las decisiones relacionadas con la migración (El Hariri, 2003; Moujoud, 2008). Estos hechos reflejan el proceso de cambio en el que está inmersa la sociedad marroquí, en la que culturalmente la toma de decisiones está claramente determinada, en primer lugar, por los hombres y, en segundo lugar, por la edad (Martín Díaz, 2008), mientras que es constatable una mayor presencia y participación de las mujeres en la sociedad y la política de Marruecos (Vallejo Peña, 2010).

Sin embargo, poco sabemos sobre los determinantes que condicionan la migración a España de personas marroquíes casadas. En lugar de considerar que el que primero en migrar lo hizo por razones económicas y el segundo -mayoritariamente la mujer, ya estuviera el varón casado o todavía soltero cuando migró- por razones familiares, en este trabajo se plantea un análisis de los factores que inciden en el calendario más o menos temprano de la reagrupación familiar de los inmigrantes marroquíes en España, diferenciando entre la perspectiva masculina y la femenina.

\section{GÉNERO, MIGRACIÓN Y MARROQUÍES}

El perfil socio-demográfico histórico de la migración marroquí —varón joven, mayoritariamente soltero, que migra solo— se ajusta, casi a la perfección, a los planteamientos clásicos sobre los determinantes de las migraciones, que han tenido como efecto no prestar una atención específica a la migración femenina, al considerar que era dependiente de la masculina y motivada por razones familiares.

Entre los planteamientos clásicos sobre las razones de las migraciones (Massey et al., 2000), probablemente la teoría neoclásica es la más importante. En ella se asume que la persona migra tras un cálculo racional del coste/ beneficio que esto le aportará. La migración implica una inversión en capital humano, ya que quien lo hace va a donde cree que, por sus características y/o habilidades personales, será más provechoso. Se trata de un planteamiento individualista, en el que quien decide es el sujeto que migra, que parece ajustarse especialmente bien al varón soltero y que asume, cuando sí hay una pareja, que ésta es un actor pasivo.

Por otro lado, en la teoría de la «nueva economía» se considera que el hogar es la unidad de decisión. En otras palabras, que cuando se produce una migración es porque se ha decidido de forma colectiva, en el seno de la 
familia, con el fin de maximizar los beneficios y reducir los riesgos, y se ha elegido a la persona que más probabilidades de éxito tiene. En esta segunda perspectiva la mujer es un miembro más del hogar y, como tal, está incluida en la decisión familiar. De alguna manera, no se le reconoce capacidad de decisión autónoma.

Ahora bien, los hogares no tienen por qué ser unidades armónicas y altruistas; en ellas también se dan conflictos de intereses, además de no contar siempre con la información más adecuada para tomar una decisión migratoria. Diversos estudios han puesto de manifiesto que en la expresión del poder, esto es, en la toma de decisiones, las mujeres son habitualmente excluidas. Frecuentemente, se aduce, la decisión de migrar no es una decisión del hogar sino del hombre dominante en el mismo. De ahí la necesidad y también el interés de contextualizar las migraciones desde la perspectiva de género (Pessar y Mahler, 2003; Silvey, 2006).

Lo cierto es que la literatura que ha analizado migraciones desde la perspectiva de género ha encontrado diferencias significativas en los determinantes de la migración de los hombres y las mujeres, especialmente relacionados con variables ligadas al ciclo de vida y a factores socio-económicos. Podemos destacar, por ejemplo, que el hecho de tener hijos condicionaba la migración femenina, mientras que no tenía efectos en el caso de los hombres²; que un nivel elevado de educación aumenta la probabilidad de migrar para las mujeres, mientras que entre los hombres la relación es cero o negativa. Poseer tierras inhibe la migración femenina pero potencia la masculina, que dispone así de algo sobre lo que pedir un crédito para viajar. Tener un negocio, por el contrario, inhibe la migración masculina pero aumenta la probabilidad de migración femenina. En general, el acceso al capital social tiene mayor importancia en la migración femenina que en la masculina, aunque el efecto es positivo en ambos casos (Geist y McManus, 2012; Heering et al., 2004; Cerrutti y Massey, 2001; De Jong, 2000).

Por otro lado, la literatura sobre migraciones desarrollada desde la perspectiva del transnacionalismo coincide en que la migración ha de ser interpretada en un contexto de vinculación dinámica de las personas inmigrantes con los mundos materiales que viven y experimentan (redes sociales y económicas, prácticas culturales). En términos generales, la primera generación de inmigrantes mantiene múltiples lazos con sus países de origen mientras se

2 González-Ferrer (2011) ha encontrado que las mujeres precursoras en la migración a España aceleran la inmigración de los hijos, y que también son más rápidas en la reagrupación de sus maridos que los hombres en la misma situación. 
adapta a las sociedades de acogida, desarrollando un interesante y complejo espacio social transnacional (Itzigsohn, J., y Giorguli-Saucedo, S., 2005). En el caso de las mujeres marroquíes, este espacio transnacional está enmarcado en un conjunto de normas culturales diferenciadas por género. Estudios como los de Salih (2001, 2003), muestran cómo las mujeres marroquíes que migraron a Italia para reunirse con sus maridos, mantienen una marcada movilidad transnacional para atender las necesidades de sus padres en Marruecos, y esto es así aún a costa de sacrificar sus aspiraciones laborales porque asumen el rol reproductivo y de cuidados esperado en la mujer.

Dos realidades inciden en que, durante mucho tiempo, las mujeres marroquíes hayan sido «invisibles» en los estudios sobre migraciones (Boukhobza, 2005). Por un lado, tradicionalmente en la sociedad marroquí el rol de la mujer la confina a la esfera estrictamente familiar y no ha sido hasta fechas muy recientes, con la aprobación de la nueva Mudawana ${ }^{3}$ en 2004, cuando ha cambiado jurídicamente su estatuto de eterna menor, lo que requería — por ejemplo— la autorización del padre o del marido para migrar. La segunda razón radicaba en la limitada oferta de empleos destinados a las mujeres en los lugares de destino, básicamente empleadas de hogar (Ouali, 2003).

Quién y por qué migra a España está condicionado por el sistema de género imperante en Marruecos y en el hogar marroquí de origen, y también por el abanico de oportunidades existente, tanto en Marruecos como en España. El funcionamiento del mercado laboral y los puestos de trabajo que brinda son factores pull de las migraciones especialmente importantes. La existencia de nichos laborales segregados por sexo perfilan las decisiones de migración y la existencia de redes específicas de apoyo. En el caso español, la presencia de hombres marroquíes en el sector primario, así como en el de la construcción y en la industria, contrasta con la presencia de mujeres marroquíes en el sector de servicios, especialmente en la categoría de cuidados a las personas. Ha sido puesto de manifiesto que el incremento de la migración femenina en el sur de Europa puede explicarse fundamentalmente por el aumento de puestos de trabajo en los sectores de servicios e informales (King y Zontini, 2000).

3 La Mudawana es el código o ley de familia que regula el matrimonio, el divorcio, la herencia y la custodia de los hijos en Marruecos. 


\section{HiPÓTESIS}

Las motivaciones para migrar son complejas y pueden estar compuestas de varias intenciones de forma simultánea, además de cambiar con el paso del tiempo y de las circunstancias personales y familiares. En este trabajo se asume que el comportamiento migratorio de las personas es un reflejo de las motivaciones que lo guían. En el caso de las personas casadas, parece lógico pensar que quien reagrupa a su familia lo hace porque considera que es lo mejor para todos y que el cónyuge reagrupado migra a España porque participa de la misma consideración. Que toda la familia nuclear viva unida en territorio español implica admitir una integración de mayor calado que, quien teniendo familia, prefiere dejarla en Marruecos. Si, además, lo hace en un plazo corto, podemos asumir que hay un mayor interés o tendencia a permanecer en España.

Por otro lado, se cree que las motivaciones para migrar no son exactamente las mismas dependiendo del sexo del migrante. Por este motivo, y centrándonos en los sujetos casados — hombres y mujeres - que tienen a su familia nuclear - cónyuge y, en un gran número de casos, a sus hijos- en España, se realiza un análisis de los determinantes de la migración marroquí diferenciando entre la perspectiva masculina y la femenina.

Junto con las motivaciones es necesario recordar que existen una serie de requisitos que establecen la posibilidad de reagrupación legal del cónyuge e hijos, que tiene unas importantes implicaciones para la vida ordinaria ${ }^{4}$. Ello no obstante, en última instancia el condicionante más importante es querer reagrupar a la familia, y nos podemos acercar a esta realidad a través del factor tiempo, o cuánto tarda la familia en estar reagrupada.

A lo largo del texto se emplea el término «reagrupación» en sentido amplio, esto es, haciendo referencia tanto a personas casadas antes de la migración

4 Según la ley española de extranjería (Ley orgánica 4/2000 del 24 de noviembre), una persona extranjera tiene derecho a solicitar la reagrupación de su cónyuge cuando lleva un año con permiso de residencia temporal y tiene permiso para estar, por lo menos, otro año más. Debe, además, acreditar que ha cotizado a la Seguridad Social al menos tres meses, tiene casa independiente y una renta suficiente para sostener al cónyuge reagrupado, incrementándose el tamaño de la casa y la renta cuando también se quiere traer a los hijos (López Hernández y Montoro Gurich, 2009). La autorización de residencia por reagrupación familiar no incluye el permiso de trabajo para el cónyuge reagrupado. Este cónyuge debe pasar dos años en el país antes de iniciar su propio proceso de obtención de permiso de residencia, desvinculándose de la autorización de reagrupación familiar que le permitió entrar y residir legalmente en el país. 
a España como a personas que migraron a España solteras y que, pasado un tiempo, volvieron a Marruecos para contraer matrimonio y terminaron trayendo a su cónyuge.

\section{FUENTE Y MÉTODOS}

La información empleada en este análisis procede del Moroccan Migration Survey, que ha entrevistado - hasta la fecha- a 262 inmigrantes marroquíes residentes en Navarra. Para ello ha adaptado la encuesta etnográfica ${ }^{5}$ del Office of Population Research de la Universidad de Princeton para el estudio de las migraciones internacionales de población latina con destino a EEUU, que se ha utilizado por primera vez en el entorno geográfico y cultural hispanomarroquí.

\section{TABLA 1}

\section{FICHA TÉCNICA DE LA ENCUESTA}

\begin{tabular}{l|l}
\hline Ámbito & \multicolumn{1}{|c}{ Comunidad Foral de Navarra } \\
\hline Universo & $\begin{array}{l}\text { Población marroquí residente en Navarra según Padrón 2013: 9.876 } \\
\text { personas }\end{array}$ \\
\hline Muestra & $\begin{array}{l}262 \text { cuestionarios en } 209 \text { hogares (residentes: 839 personas, 8,5\% } \\
\text { población de origen marroquí empadronada en Navarra). 209 dirigidos } \\
\text { a «jefes de hogar» y 53 a «cónyuges jefes de hogar» }\end{array}$ \\
\hline Muestreo & $\begin{array}{l}\text { Ajustado a la distribución geográfica de la población marroquí: } \\
\text { Pamplona y comarca, 37\% cuestionarios. Ribera, 63\% cuestionarios. En } \\
\text { ambos escenarios el 80\% de las encuestas se ha realizado a «jefes de } \\
\text { hogar» y el 20\% restante a «cónyuges jefes de hogar». Se han utilizado } \\
\text { diversas formas de contacto: amistad, vecindad, mezquita, asociaciones, } \\
\text { viandantes, Cruz Roja, colaboraciones en otros estudios, elaboración de } \\
\text { listados propios de establecimientos y comercios }\end{array}$ \\
\hline Tipología & Entrevista personal, realizada principalmente en el hogar \\
\hline $\begin{array}{l}\text { Fechas del trabajo } \\
\text { de campo }\end{array}$ & $\begin{array}{l}\text { Pilotaje: } 21 \text { al 27 de Octubre de 2013. Resto: Del 11 de Noviembre al } \\
10 \text { de Diciembre de 2013 }\end{array}$ \\
\hline Entrevistadores & 6 personas de origen marroquí, 3 mujeres y 3 hombres \\
\hline Empresa & CIES. Estudios de opinión y de mercado \\
\hline
\end{tabular}

5 La versión más actualizada de la encuesta etnográfica, que es la que se ha adaptado, puede consultarse en: mmp.opr.princeton.edu/research/questionnaire-es.aspx 
Se ha utilizado el Padrón para localizar a la población inmigrante nacida en Marruecos y residente en los municipios de Navarra, y decidir el número de hogares que debían ser entrevistados, fijándose este número en 209 hogares. La labor de campo se desarrolló entre finales de octubre y comienzos de diciembre de 2013.

El cuestionario se caracteriza porque tiene un formato semiestructurado que facilita la realización de una entrevista flexible pero con información idéntica para todos los entrevistados. La encuesta se realiza al jefe de hogar, quien proporciona información sobre todas las personas con las que convive en el hogar y, de forma significativamente más extensa, sobre su cónyuge o pareja y sobre sí mismo. Además de obtener datos socio-demográficos y familiares del sujeto y de los miembros de su hogar, se consigue una completa visión sobre la historia migratoria de todas las personas del hogar, además de una historia de vida laboral detallada sobre el sujeto y su cónyuge, la acumulación de recursos, el uso de servicios sociales y las condiciones de salud actual y previa a la migración.

Entre los marroquíes, el hombre es culturalmente el jefe de hogar -incluso cuando está ausente- y sólo de forma puntual encontramos mujeres que ocupan este lugar - mayoritariamente, viudas y divorciadas-. De los 209 jefes de hogar, 177 eran hombres frente a 32 mujeres. Con el fin de captar una perspectiva más amplia de los procesos migratorios marroquíes, población que se caracteriza por unos patrones de género muy diferenciados (Martín Díaz, 2008; Pels y De Haan, 2007; Soriano Miras, 2008), se decidió realizar también encuestas a mujeres casadas ya que, al convivir con su marido, en otro caso sólo se dispondría información sobre ellas a través de sus parejas. Por este motivo, la muestra final de encuestas incluye a 53 esposas de varones jefes de hogar, llegándose a un total de 262 entrevistas. Este trabajo emplea las encuestas realizadas a personas casadas en el momento de la entrevista, un total de 165 varones (un 93,3\% de los cuales se definen «jefe de hogar») y de 64 mujeres (11 de las cuales, el 17,2\%, se definen «jefe de hogar»).

Otra variable que ayuda a definir nuestra población es la clasificación de cada sujeto casado según la relación entre el momento de la migración y el de formación de la familia. El perfil mayoritario en las dos muestras trabajadas coincide con el clásico de migración marroquí: los varones son precursores de la migración. Un $60 \%$ emigró soltero, y volvió para buscar pareja en una compatriota, mientras que un $25 \%$ de la muestra ya estaba casada y terminó reagrupando a la esposa. Sin embargo, es interesante señalar que en la muestra de las mujeres, junto con el esperable perfil de esposa reagrupada -más de un $60 \%$ de la muestra- aparece un perfil novedoso, el de la mujer precursora de 
la migración, con casi un tercio de la muestra. Este hecho refleja la apertura por parte de las mujeres y la sociedad marroquí a la migración femenina autónoma y produce la diversificación de los perfiles migratorios a la que se hacía referencia en la introducción ${ }^{6}$.

La información original de la encuesta ha sido trabajada, recodificando las categorías existentes en nuevas variables para ajustar mejor el análisis de acuerdo con nuestros objetivos. Para testar la hipótesis de partida se ha creado la variable dependiente que hemos llamado «reagrupación temprana», cuyos valores son 1, cuando la reagrupación familiar se ha producido en 6 o menos años, y 0 , cuando la reagrupación familiar se ha producido después de 6 o más años ${ }^{7}$. La lectura de esta variable sería que, cuando la reagrupación es temprana, estas personas tienen un mayor interés o tendencia a permanecer en España ${ }^{8}$. A continuación se pensaron posibles factores que podían explicar la reagrupación del cónyuge que, con información de la encuesta, han sido plasmados en variables independientes.

Con el fin de comprobar la validez del modelo en el que las variables independientes predicen la reagrupación acelerada o no acelerada, se realizó un análisis de regresión logística binaria. Este tipo de análisis multivariante permite predecir o evaluar la relación entre la variable dependiente dicotómica (agrupación acelerada o agrupación no acelerada) con otra $u$ otras variables independientes y de control. En nuestro caso, analizamos la probabilidad de que una reagrupación sea acelerada $(\mathrm{Y}=1)$ como función de las $\mathrm{X}$ variables independientes: $\mathrm{X} 1$ : «nivel de estudios»; X2: número de hijos pequeños; X3: red familiar en España, etc.

La ecuación general (función logística) que se aplica para los dos grupos analizados es:

$$
P(Y=1)=\frac{1}{1+\exp \left(-\beta_{o}-\beta_{1} \cdot X_{1}-\beta_{2} \cdot X_{2} \cdot-\beta_{3} \cdot X_{3}\right)}
$$

6 Sin embargo, no ha sido posible constatar diferencias significativas en los niveles educativos, las características de los hogares o la participación laboral de las mujeres dependiendo de la relación entre el momento de la migración y la formación de la familia, señalados recientemente por González-Ferrer (2011).

7 El rango temporal de reagrupación en la población entrevistada oscila entre 0 años —vinieron juntos- y 25 años. Aproximadamente un 50\% de los marroquíes consiguieron reagrupar a su familia en seis o menos años. El concepto de reagrupación empleado en este trabajo es amplio e incluye a todas las parejas reunidas independientemente de su situación legal.

8 Decimos España, y no Navarra, porque la población marroquí entrevistada aunque reside en la actualidad en la Comunidad Foral no ha tenido por qué ser así desde el principio de su llegada, tanto de ego como del cónyuge reagrupado. 
Con base en los dos grupos de datos de estos años y mediante el uso de técnicas de regresión logística por pasos, podemos conocer qué variables tienen mayor o menor influencia en la reagrupación acelerada. El objetivo perseguido con esta técnica es expresar la probabilidad de agrupación acelerada como función de las $\mathrm{X}$ variables, que se presumen relevantes o influyentes (cuanto más cercano a 0 fuera el valor sig., más probabilidad tenía una reagrupación de ser más rápida).

Las variables incluidas en el análisis son: edad de cada uno de los cónyuges en el momento de la reagrupación, tiempo que lleva el cónyuge reagrupado en España con respecto al año 2008 (cuando es más clara la situación de crisis económica); variables relativas al capital social del sujeto como: número de hijos pequeños nacidos antes de la reagrupación ${ }^{9}$, autodefinición como jefe de hogar (si o no), existencia de red familiar del jefe de hogar en España ${ }^{10}$; nivel de estudios completado de la persona entrevistada y de su cónyuge, clasificado en dos categorías: nivel bajo (personas con nivel de primaria o menos) y nivel alto (personas con estudios de secundaria/FP o universitarios); actividad laboral (tenían o no trabajo) de la persona entrevistada y de su cónyuge en el año de la llegada del cónyuge; opinión (positiva o negativa) ante el trabajo de la mujer fuera del hogar ${ }^{11}$; variables relativas al capital físico de la persona entrevistada y de su cónyuge como son posesión de vivienda y posesión de un negocio, ambos en España. La tabla 2 presenta la descripción de estas variables.

9 Definiendo como "hijos pequeños" a aquellos que tenían menos de 6 años, edad en la que empieza la enseñanza obligatoria en el sistema educativo español.

10 Se trata de una variable que, tras localizar si el jefe de hogar tenía al menos un pariente directo en España (padre, madre, hermano/s o hermana/s, principalmente), los clasifica entre aquellos que sí tienen red (uno o más familiares) frente a aquellos que no la tienen.

11 En las encuestas realizadas a mujeres, las casadas jefes de hogar consideran que la mujer puede trabajar fuera del hogar en un $100 \%$ de los casos. En el de las mujeres casadas que no son jefes de hogar la respuesta está mediatizada porque responden según lo que creen que piensan sus maridos y, sin embargo, en el 96,2\% de los casos la respuesta es positiva. Tan sólo un 3,8\% creen que sus maridos están en contra del trabajo femenino fuera del hogar. 
TABLA 2

DESCRIPCIÓN DE LAS VARIABLES INDEPENDIENTES EN LAS DOS MUESTRAS

\begin{tabular}{|c|c|c|}
\hline Variables independientes & Varones & Mujeres \\
\hline & $S^{2}(S)$ & $S^{2}(S)$ \\
\hline Edad de ego cuando la reagrupación & $36,751(7,690)$ & $31,03(7,954)$ \\
\hline Edad del cónyuge cuando la reagrupación & $30,342(8,179)$ & $38,27(7,769)$ \\
\hline Años reagrupados en España antes de 2008 & $9,6(5,723)$ & $8,5(5,048)$ \\
\hline $\mathrm{N}^{o}$ hijos pequeños antes de reagrupación & $0,51(0,73)$ & $0,68(0,69)$ \\
\hline Nivel estudios varón & $\%$ & $\%$ \\
\hline Bajo (hasta primaria) & 55,1 & 57,8 \\
\hline Alto (desde secundaria) & 44,9 & 42,2 \\
\hline \multicolumn{3}{|l|}{ Nivel estudios mujer } \\
\hline Bajo (hasta primaria) & 58,0 & 51,6 \\
\hline Alto (desde secundaria) & 42,0 & 48,4 \\
\hline \multicolumn{3}{|l|}{ Jefe de hogar } \\
\hline No & 5,1 & 82,8 \\
\hline Sí & 94,9 & 17,2 \\
\hline \multicolumn{3}{|l|}{ Red familiar del varón en España } \\
\hline No & 53,4 & 57,8 \\
\hline Sí & 46,6 & 42,2 \\
\hline \multicolumn{3}{|l|}{ Trabajo del varón en el año de la reagrupación } \\
\hline No tiene & 6,3 & 7,8 \\
\hline Sí tiene & 93,8 & 92,2 \\
\hline \multicolumn{3}{|l|}{ Trabajo de la mujer en el año de la reagrupación } \\
\hline No tiene & 75,8 & 72,2 \\
\hline Sí tiene & 24,2 & 27,8 \\
\hline \multicolumn{3}{|l|}{ Trabajo femenino fuera del hogar } \\
\hline En contra & 23,3 & 3,2 \\
\hline A favor & 76,7 & 96,9 \\
\hline \multicolumn{3}{|l|}{ Negocio propio } \\
\hline No tiene o lo tuvo posterior a reagrupación & 93,1 & 93,4 \\
\hline Lo puso antes de reagrupación & 6,9 & 6,6 \\
\hline \multicolumn{3}{|l|}{ Vivienda en propiedad } \\
\hline No propia, o comprada después reagrupación & 93,8 & 93,4 \\
\hline \multirow[t]{2}{*}{ Comprada antes reagrupación } & 6,2 & 6,6 \\
\hline & $\mathrm{N}=165$ & $\mathrm{~N}=64$ \\
\hline
\end{tabular}

Fuente: Moroccan Migration Survey.

Estudios Geográficos, Vol. LXXVIII, 283, pp. 445-464, julio-diciembre 2017

ISSN: 0014-1496, eISSN: 1988-8546, doi: 10.3989/estgeogr.201715 
Se han ejecutado modelos separados por sexo, considerando los mismos factores o variables independientes. Las pruebas de ajuste global (pruebas ómnibus y contrastes de Hosmer y Lemeshow) abogan por la significatividad del modelo final. El modelo final de los hombres explica entre un 0,30\% (prueba de ajuste de Cox y Snell) y un 0,41\% (prueba de ajuste de Nagelkerke) y el modelo de las mujeres explica entre un 0,45\% (prueba de ajuste de Cox y Snell) y un 0,73\% (prueba de ajuste de Nagelkerke) de la relación entre la probabilidad de ocurrencia de la reagrupación acelerada y las variables explicativas, lo cual es considerado un porcentaje aceptable de la bondad de ambos modelos. Con base en estos datos, podemos observar qué variables independientes explicaban el que una agrupación sea acelerada (tablas 3 y $4^{12}$ ).

\section{RESUlTAdOS Y DISCUSIÓN}

La lógica de las variables introducidas en el análisis se presenta ahora, enfrentándola a los resultados obtenidos. Así, con respecto a las variables asociadas a capital humano, sería esperable que a más edad de los sujetos en el año de la reagrupación- más probable fuera la reagrupación temprana, asumiendo un mayor control de recursos y más contactos en la sociedad española. Por otro lado, quizá entre los sujetos de más edad cabría encontrar personas menos favorables a la reagrupación, o menos susceptibles a la integración en España. En cuanto al nivel de estudios, es de suponer que cuanto más alto fuera el nivel de estudios de ego, más fácil le fuera la incorporación al mercado laboral y, por tanto, una más temprana reagrupación familiar. Sin embargo, conociendo cuál es el nicho laboral de los marroquíes, en sectores donde la formación no es imprescindible, parece también que podamos esperar que no sea muy significativo. Los resultados muestran que estas dos variables —edad y nivel de estudios - no son significativas en el modelo de los hombres (tabla 3), mientras que entre las mujeres no es significativo el nivel de estudios pero sí lo es la edad que tenía el marido en el momento de la reagrupación, aunque la fuerza explicativa de esta variable sea pequeña

12 Estas tablas presentan únicamente las variables independientes significativas $(\mathrm{Sig} \leq, 05)$ en el análisis de regresión para cada uno de los sexos. En otras palabras, aquellas que inciden en la reagrupación temprana o no de los marroquíes de nuestra muestra. Un valor superior a 1 de $B$ indica que la variable independiente considerada incrementa la respuesta (reagrupación temprana, en nuestro caso) y un valor inferior a 1 de B (o negativo) que la disminuye. El "odds ratio" muestra la fuerza de la relación. Cuanto más alto sea, más importante es la variable considerada a la hora de explicar la reagrupación temprana. 
$(B=0,405$, odds ratio $=1,500$ : cuando el marido tiene una edad relativamente elevada, la probabilidad de realizar una reagrupación temprana es 1,5 veces mayor que cuando se trata de un varón relativamente joven) (tabla 4).

TABLA 3

HOMBRES: MODELO MULTIVARIANTE DE PROBABILIDAD

DE REAGRUPACIÓN TEMPRANA

\begin{tabular}{l|c|c}
\hline Variable en el modelo & $\begin{array}{c}\text { Coeficiente } \\
\text { B }\end{array}$ & $\begin{array}{c}\text { Interpretación de B } \\
\text { (odds ratio) }\end{array}$ \\
\hline $\begin{array}{l}\text { Trabajo de la mujer en el año de la } \\
\text { reagrupación }\end{array}$ & $1,842^{*}$ & $\begin{array}{c}6,307 \\
\text { (veces que puede afectar) }\end{array}$ \\
\hline Trabajo femenino fuera del hogar & $-1,208^{*}$ & $\begin{array}{c}, 299 \\
\text { (afecta moderadamente) }\end{array}$ \\
\hline Años reagrupados en España antes 2008 &,$- 278^{*}$ & $\begin{array}{c}, 757 \\
\text { (afecta moderadamente) }\end{array}$ \\
\hline
\end{tabular}

(*) significativo a un $5 \%$

TABLA 4

MUJERES: MODELO MULTIVARIANTE DE PROBABILIDAD

DE REAGRUPACIÓN TEMPRANA

\begin{tabular}{|c|c|c|}
\hline Variable en el modelo & $\begin{array}{c}\text { Coeficiente } \\
\text { B }\end{array}$ & $\begin{array}{l}\text { Interpretación de B } \\
\text { (odds ratio) }\end{array}$ \\
\hline Edad del marido &, $405^{*}$ & $\begin{array}{c}1,5 \\
\text { (veces que puede afectar) }\end{array}$ \\
\hline $\begin{array}{l}\text { Núm. de hijos pequeños antes } \\
\text { reagrupación }\end{array}$ & $-2,171$ & $\begin{array}{c}, 114 \\
\text { (apenas afecta) }\end{array}$ \\
\hline $\begin{array}{l}\text { Trabajo de la mujer en el año de la } \\
\text { reagrupación }\end{array}$ & $4,995^{*}$ & $\begin{array}{c}147,610 \\
\text { (veces que puede afectar) }\end{array}$ \\
\hline Jefe de hogar & $-10,724 *$ & $\begin{array}{c}, 000 \\
\text { (no afecta) }\end{array}$ \\
\hline Años reagrupados en España antes 2008 &,$- 577^{*}$ & $\begin{array}{c}, 562 \\
\text { (veces que puede afectar) }\end{array}$ \\
\hline
\end{tabular}

(*) significativo a un $5 \%$ 
En lo que a las variables relacionadas con el capital social se refiere, sería esperable según la literatura que la presencia de hijos condicionara la migración femenina. Especialmente, en nuestra opinión, si estos hijos son pequeños y requieren, por lo tanto, de un mayor cuidado y dedicación, tanto más en un entorno diferente. Lo lógico sería que el hecho de tener hijos por debajo de la edad de la escolarización obligatoria actuara como un factor en contra de la reagrupación temprana en el modelo de las mujeres, mientras que cabía esperar que no fuera significativo en el modelo de los hombres. Los resultados, sin embargo, no son exactamente los esperados: en el caso de los varones la presencia de hijos pequeños no es, en efecto, significativa; en el de las mujeres esta variable, no siendo significativa, se encuentra en el límite y, además, apunta en el sentido de lo que se esperaba $(B=-2,171$, Sig 0,061 , odds ratio $=0,114$, tabla 4$)$. El coeficiente negativo está indicando que con un número menor de niños pequeños la decisión de reagrupar se acelera frente a una situación con un número mayor de niños pequeños. Podemos asumir que, cuantos menos niños pequeños tiene la familia, mayor libertad de movimientos puede tener la mujer en España.

Otra variable de este grupo relacionada con capital social es si la persona encuestada es o no jefe del hogar. Admitiendo que se trata de generaciones en las que la consideración de ser o no jefe de hogar expresa un papel desigual en la toma de decisiones, parece lógico que ostentar dicha jefatura tendrá efectos directos sobre la reagrupación temprana del cónyuge. Sin embargo, en el modelo masculino esta variable no tiene significación a la hora de explicar la reagrupación temprana, mientras que en el modelo de las mujeres sí es significativa $y$, además, presenta un coeficiente negativo $(B=-10,724$, odds ratio $=0,000$ ) (tabla 4). Esto está indicando que cuando la mujer no es jefe de hogar, la decisión de reagrupar se acelera frente a la situación en la que la mujer es jefe de hogar. En otras palabras, entre los marroquíes la decisión de reagrupar a la familia depende, principalmente, del varón. Sin embargo, como el odds ratio es 0 , la probabilidad de agrupación no se ve alterada por esta variable.

Finalmente, la variable de existencia de red familiar del jefe de hogar en España no es significativa en ninguno de los dos modelos; tener familiares directos en España no tiene efectos en la reagrupación temprana de las personas marroquíes.

Las variables relacionadas con el trabajo muestran una realidad muy interesante. El trabajo del varón no tiene significación estadística en ninguno de los dos modelos. Aunque mayoritariamente el hombre cuente con trabajo situación necesaria, desde el punto de vista legal, y aconsejable, podríamos decir, desde el punto de vista práctico-, no es un factor decisivo a la hora de explicar una reagrupación temprana. Sin embargo, el trabajo de la mujer en España en el mismo año de la reagrupación sí es una variable significativa, tanto desde el punto 
de vista de los hombres como desde las mujeres. Podemos interpretar que, o bien la mujer ha sido reagrupada con un trabajo a la vista o bien, una vez reagrupada, ha encontrado trabajo rápidamente. El hecho es que su acceso - legal o no ${ }^{13}-$ a empleos de un nicho laboral muy característico ${ }^{14}$ es el factor predictor de la reagrupación temprana más importante para los dos sexos; aumenta en más de 6 veces la probabilidad de la reagrupación tem-prana en el modelo de los hombres ( $B=1,842$, Sig 0,000 , odds ratio $=6,307$, tabla 3 ) y en más de 147 veces en el de las mujeres $(B=4,995$, Sig 0,006 , odds ratio $=147,610$, tabla 4$)$.

Otra variable relacionada con trabajo es la consideración, por parte del jefe de hogar, de si la mujer puede o no trabajar fuera de casa. La lógica sería que si la respuesta es positiva, la reagrupación fuera temprana por cuanto implícitamente se estaría contando con el apoyo económico de un segundo sueldo en España. Esta variable es significativa en el modelo de los hombres y, además, de signo negativo, lo que indica que aún en los casos en los que se considera que las mujeres no pueden trabajar fuera de casa se ha producido la reagrupación temprana; en otras palabras, parece que la necesidad económica se ha impuesto al planteamiento ideológico $(B=-1,208$, Sig 0,048, odds ratio=0,299, tabla 3).

En el modelo de las mujeres esta variable no tiene significación, quizá porque las mujeres jefes de hogar consideran, al $100 \%$, que la mujer sí puede trabajar fuera del hogar y, entre las mujeres que no son jefes de hogar, la inmensa mayoría (un 96,2\%) cree que el jefe de hogar — su marido— está a favor del trabajo femenino fuera del hogar. Sin embargo, esta respuesta contrasta claramente con la realidad de la contestación masculina, en la que un importante $24,8 \%$ está en contra del trabajo de la mujer fuera del hogar (tabla 2). Pareciera que entre las mujeres se interpreta que el trabajo femenino fuera del hogar, al ser necesario para la familia, no está mal visto y se acepta por parte de los hombres. Sin embargo, entre los varones, más bien parece que se pliegan a la realidad económica, conservando intacto el sentimiento de desagrado ante el trabajo femenino fuera del hogar.

Estos datos parecen apuntar a que en estas parejas no se ha producido un proceso de toma de decisión explícito sino más bien implícito, ad hoc, ajustado a

13 El permiso que se concede a la mujer por reagrupación es temporal, por un año y renovable, y no le da derecho a trabajar. La mujer reagrupada puede obtener una autorización de residencia temporal cuando obtenga la autorización para trabajar (siempre que las condiciones del contrato de trabajo sean las adecuadas), haya residido en España durante cinco años y no medie separación matrimonial (López Hernández y Montoro Gurich (dirs.), 2009, pág. 31 y sig.).

14 La ocupación principal de la mujer reagrupada en el año de la reagrupación era, en un $61,4 \%$, ama de casa, un $14 \%$ estaba desempleada y, entre las que trabajaban fuera del hogar, un $9,1 \%$ eran empleadas domésticas, un 4,5\% estaban en la industria alimentaria, un 2,3\% eran ayudantes de cocina y otro $2,3 \%$ peones agrícolas. 
la situación concreta, evitando el conflicto. Se llega así a un acuerdo silencioso, característico de familias en las que los roles de género no se desvían de los planteamientos tradicionales (Wiesmann et al., 2008).

Con respecto a las variables relacionadas con el capital físico, ya que los marroquíes encuestados en Navarra no poseen parcelas de tierra, se ha elegido la posesión de una vivienda y la de un negocio. La lógica es que la compra de una vivienda previa a la reagrupación invitaría a que ésta fuera temprana, con el objetivo de contar con el apoyo para su pago del eventual trabajo del cónyuge. Asimismo, en el caso del negocio y considerando que éste está radicado en España, una reagrupación temprana supondría contar con ayuda familiar en el mismo. Pues bien, en ambos casos el porcentaje de marroquíes que compraron o pusieron un negocio previo a la reagrupación de su cónyuge es muy reducido y el análisis multivariante muestra que estos factores no han sido significativos a la hora de aumentar la probabilidad de una migración rápida a través de la reagrupación en ninguno de los dos modelos.

La última variable presente en ambos modelos es la del intervalo de años de la reagrupación con respecto a 2008, el año más intenso de crisis económica. La lógica esperable sería encontrar una reagrupación temprana cuanto mayor sea dicho intervalo, esto es, que el cónyuge fuera reagrupado en época de bonanza en la que, presumiblemente, las posibilidades laborales y de ahorro serían mayores. Sin embargo este factor, significativo en los dos modelos aunque con poca capacidad predictiva, tiene signo negativo (varones, $\mathrm{B}=-0,278$, odds ratio $=0,757$, tabla 3; mujeres, $B=-0,577$, odds ratio $=0,562$, tabla 4); su interpretación es que cuanto más corto es el intervalo, más temprana ha sido la reagrupación. Esto es, cuanto peor es la situación económica y con más fuerza está golpeando la crisis económica, antes se realiza la reagrupación, posiblemente para contar con el apoyo de los ingresos de un eventual trabajo del cónyuge.

\section{CONCLUSIONES}

Este trabajo plantea un análisis por género de los determinantes de la migración de personas marroquíes casadas, centrándose en un tipo muy concreto de migración caracterizado por su especificidad: el de la reagrupación del cónyuge, que en términos de migración marroquí consiste mayoritariamente en varones reagrupando a mujeres.

Se partía de la hipótesis de que una reagrupación familiar temprana implica una integración de mayor calado que quien teniendo familia, prefiere dejarla en Marruecos o reagruparla tardíamente, quizá porque no ha podido soportar 
más las presiones familiares en el sentido de que quieren vivir en España. Empleando información proveniente de la encuesta etnográfica desarrollada en el último trimestre de 2013 en Navarra y un análisis de regresión multivariante, se estudia qué variables independientes predicen una reagrupación acelerada (en menos de seis años) o no acelerada (más de seis años) para cada sexo.

El resultado de los modelos multivariantes refleja una realidad marcada por las motivaciones económicas; el factor que más explica una reagrupación temprana es el trabajo de la mujer en el mismo año de la reagrupación, y las otras variables explicativas abundan en esta perspectiva: la cercanía del año de la reagrupación al año de máxima crisis económica en España -2008- para los dos sexos, y en los hombres la actitud ante el trabajo de la mujer fuera del hogar. Entre las mujeres aparece, como un elemento relacionado con el ciclo vital, el hecho de tener hijos pequeños antes de la reagrupación —un factor con claras implicaciones sobre la disponibilidad femenina en el mercado de trabajo- y otro par de variables que también nos hablan de la importancia de la esfera de lo privado: la edad del varón-cónyuge en el momento de la reagrupación y el hecho de ser, o no, jefe de hogar. En cualquier caso, y dados los coeficientes explicativos de estas variables en el modelo de las mujeres, la reagrupación familiar de la migración marroquí con destino a España tiene, para los dos sexos, una lectura en clave laboral muy marcada.

\section{Agradecimientos}

Este trabajo se inscribe en el proyecto titulado "Migración marroquí en España: perspectivas de origen y destino", que se inició en 2011 fruto del convenio entre la Universidad de Navarra, la Universidad de Princeton (Office of Population Research) y Cruz Roja Navarra. Ha recibido subvenciones del Gobierno de Navarra (Convocatorias Jerónimo de Ayanz 2011 y 2012) y de la Fundación Universitaria de Navarra (2013 y 2014).

Una versión preliminar de este trabajo se presentó en el XIV Congreso Nacional de Población (Sevilla, 2014).

\section{BibliograFÍA}

Boukhobza, N. (2005): "Les filles naissent après les garcons". Revue européenne des migrations internacionales [en ligne], 21, 1. http://remi.revues.org/2333; DOI: 104000/remi/2333, consultado el 12/08/2013. 
Cebolla Boado, H.; Requena Díez de Revenga, M. (2009): "Los inmigrantes marroquíes en España” en Reher Sullivan, D.S.; Requena Díez de Revenga, M. (eds.), Las múltiples caras de la emigración en España, Alianza, Madrid, 251-287.

Cebolla Boado, H.; González Ferrer, A. (2008): La inmigración en España (20002007). De la gestión de flujos a la integración de los inmigrantes, Centros de Estudios Políticos y Constitucionales, Madrid.

Cerrutti, M.; Massey, D. (2001): "On the auspices of Female Migration from Mexico to the United States", Demography, 38, 2, 187-200.

de Haas, H. (2005): Morocco: From emigration country to Africa's migration passage to Europe, Migration Policy Institute, Washington.

de Haas, H. (2007): "Morocco's Migration Experience: A Transitional Perspective". International Migration, 45, 4, 39-70.

De Jong, G.F. (2000): "Expectations, Gender and Norms in Migration DecisionMaking". Population Studies, 54, 3, 307-319.

El Hariri, S. (2003): "Les femmes et le retour au pays d'origine". Hommes et migrations, $1242,43-52$.

Geist, C.; McManus, P. (2012): "Different reasons, different results: Implications of migration by gender and family status". Demography, 49, 197-217.

González-Ferrer, A. (2011): "Explaining the labour performance of immigrant women in Spain: The interplay between family, migration and legal trajectories". International Journal of Comparative Sociology, 52, 1-2, 63-78.

Heering, L.; van der Erf, R.; van Wissen, L. (2004): "The role of family networks and migration culture in the continuation of Moroccan emigration: a gender perspective". Journal of Ethnic and Migration Studies, 30, 2, 323-337.

Izquierdo Escribano, A.; León-Alfonso, S. (2008): "La inmigración hacia dentro: argumentos sobre la necesidad de la coordinación de las políticas de inmigración en un Estado multinivel". Política y Sociedad, 45, 1, 11-39.

Itzigsohn, J.; Giorguli-Saucedo, S. (2005): "Incorporation, Transnationalism, and Gender: Immigrant Incorporation and Transnational Participation as Gendered Processes". International Migration Review, 39, 4, 895-920.

King, R.; Zontini, E. (2000): "The role of gender in the South European immigration model". Papers, 60, 35-52.

López Hernández, D.; Montoro Gurich, C. (dirs.) (2009): La reagrupación familiar de personas extranjeras en Navarra. Pamplona, Gobierno de Navarra, Oficina de Atención a la Inmigración. Extraído 15/10/2012 de: <http://www.navarra.es/NR/ rdonlyres/0BD6BB88-1388-498D-806F-0E7949D20CB5/165308/Reagrupacion_ familiar_en_Navarra.pdf>

Martín Díaz, E. (2008): "El impacto de género en las migraciones de la globalización: mujeres, trabajos y relaciones interculturales". Scripta Nova, XII, 270 (133).

Massey, D.; Arango, J.; Graeme, H.; Kouaouci, A.; Pellegrino, A.; Taylor, E. (2000): "Teorías sobre la migración internacional: una reseña y una evaluación". Trabajo, 3, 15-30. 
Massey, D.; Espinoza Higgins, M. (2014): "Moroccan Immigrants in Spain and the United States: A Comparative Analysis”. AA.VV., Emigración, identidad y países receptores, Tirant lo Blach, Valencia, 31-50.

Montoro Gurich, C. (2014): "Inmigrantes marroquíes en España: transformaciones recientes en los perfiles socio-demográficos". AA.VV., Emigración, identidad y países receptores, Tirant lo Blach, Valencia, 31-50.

Moujoud, N. (2008): "Effects de la migration sur les femmes et sur les rapports sociaux de sexe. Au-delà des visions binaires". Les cahiers du CEDREF, 16, 56-79. URL:http://cedref.revues.org/577, consultado 24/10/2013.

Ouali, N. (2003): "Les Marocaines en Europe: diversification des profiles migratoires". Hommes et migrations, 1242, 71-82.

Pels, T.; de Haan, M. (2007): "Socialization practices of Moroccan families after migration: A reconstruction in an 'acculturative arena'". Young, 15, 1, 72-89.

Pessar, P.R.; Mahler, S.J. (2003): "Transnational Migration: Bringing Gender In". International Migration Review, 37, 3, 812-846.

Reinaldo Espinosa, M. (2007): "La dinámica migratoria internacional. Reflexiones sobre el caso de la mujer magrebí en Europa". Entelequia. Revista Interdisciplinar, 4, 351381. http://www.eumed.net/entelequia/es.art.php?a=04a19, extraído 15/10/2012.

Salih, R. (2001): "Moroccan migrant women: transnationalism, nation-states and gender". Journal of Ethnic and Migration Studies, 27, 4, 655-671.

Salih, R. (2003): Gender in transnationalism: home, longing and belonging among Moroccan migrant women, Routledge, London \& New York.

Silvey, R. (2006): "Geographies of Gender and Migration: Spatializing Social Difference". International Migration Review, 40, 1, 64-81.

Soriano Miras, R. (2006): "La inmigración femenina marroquí y su asentamiento en España. Un estudio desde la Grounded Theory". Revista de Investigaciones Sociológicas, RIS, LXIV, 43, 169-191.

Soriano Miras, R. (2008): "Inmigración e identidad social: similitudes y diferencias en el proyecto migratorio de mexicanas a EEUU y mujeres marroquíes a España". Migraciones, 23, 117-150.

Vallejo Peña, F. (2010): "Evolución reciente de la participación política y social en la mujer marroquí: un análisis a través de la encuesta mundial de valores (20012007)". Revista Internacional de Pensamiento Político, 5, 287-306.

Wiesmann, S.; Boeije, H.; van Doorne-Huiskes, A.; den Dulk, L. (2008): "Not worth mentioning: The implicit and explicit nature of decisión-making about the división of paid and domestic work". Community, Work E Family, 11, 4, 341-363.

Fecha de recepción: 8 de septiembre de 2015 .

Fecha de aceptación: 27 de abril de 2016. 